\title{
Minimally invasive approach to resection of paraspinal schwannoma
}

\author{
Patrick Paullus, MD, Taylor A. Wilson, MD, Paul Lee, MD, Arunprasad Gunasekaran, BS, \\ and Noojan Kazemi, MD
}

Department of Neurosurgery, University of Arkansas for Medical Sciences, Little Rock, Arkansas

In this video, the authors demonstrate a minimally invasive approach and resection of a paraspinal schwannoma. Using an expandable retractor, the authors were able to identify important adjacent bony landmarks and hence visualize and remove this peripheral nerve sheath tumor. While a tubular retractor is commonly used for interbody fusion procedures, the location of the tumor allowed this minimally invasive approach resulting in excellent access, minimal soft-tissue injury, and a short hospital stay. The authors present this approach as a less invasive and yet effective technique for resection of otherwise difficult-to-access nerve lesions.

The video can be found here: https://youtu.be/890Y5wdMB_k.

KEYWORDS paraspinal; peripheral nerve sheath; schwannoma; minimally invasive spine; tubular retractor; video 